\title{
Comparison of Peripheral Blood Films Stained by Giemsa Stain, Rapid Diagnostic Tests for Antigen and Antibody for Detection of Plasmodium vivax and Plasmodium falciparum in Clinically Suspected Cases of Malaria
}

\author{
Sangita Vasava*, Sucheta Lakhani, Jitendra Lakhani and Radhika Khara
}

Department of Microbiology, SBKS MI \& RC, Piparia, Waghodia-391760, Gujarat, India

*Corresponding author

\begin{tabular}{|l}
\hline Ke y w o r d s \\
Giemsa staining, \\
Malaria, Rapid \\
Diagnostic Tests \\
(RDTs)
\end{tabular}

\section{Introduction}

Malaria is an infection caused by Plasmodium parasites. The parasites are spread to people through the bites of infected female Anopheles mosquitoes, called "malaria vectors." $P$. falciparum is the most prevalent malaria parasite on the African continent. It is responsible for severe clinical manifestations and most malaria-related deaths globally. $P$. vivax is the dominant malaria parasite in most countries outside of sub-Saharan Africa in WHO Malaria key facts, 11 June 2018. The WHO African Region continues to account for about $90 \%$ of malaria cases and deaths worldwide. Fifteen countries - all but one in sub-Saharan Africa - carry $80 \%$ of the global malaria burden in the World malaria report 
2017. Malaria is one of the major public health challenges and syndromic approach is unreliable because of non-specific and overlapping symptoms with other febrile diseases (Prakriti et al., 2014). The disease is a protozoan infection of red blood cells transmitted by the bite of a blood-feeding female Anopheline mosquito (Gordon et al., $2009,22^{\text {nd }}$ ed.)

Four species of malaria parasites were capable of infecting humans: P.falciparum, P.vivax, P.ovale and P.malariae; however recently a fifth P.knowlesi has been recognized as a significant human pathogen (Alan et al., 2012, $9^{\text {th }}$ ed). Various diagnostic techniques available are including: Peripheral blood film examination, rapid diagnostic tests for antigen and one step Pf/Pv antibody detection.

Present study was conducted to find out sensitivity and specificity of serodiagnostic tests, that is commercially available to correlate Giemsa based microscopy, pf HRP-2 antigen test, pan malaria pLDH and malaria antibody test.

The overlapping of malaria symptoms with other tropical diseases impairs diagnostic specificity, which can promote the indiscriminate use of antimalarials and compromise the quality of care for patients with non malarial fevers in endemic areas (Mwangi et al., 2005, Reyburn et al., 2004, McMorrow et al., 2008). The accuracy of malaria diagnosis can be greatly enhanced by combining clinical and parasite based findings (Kyabayinze et al., 2008). The standard method of malaria diagnosis is by the microscopic examination of Giemsa-stained blood smears (Rosenthal et al., 2012). Expert microscopy gives information about parasite stage and parasitaemia. Rapid diagnostic tests (RDTs) that detect malaria parasite proteins by immunochromatography have been used as complementary detection methods for malaria diagnosis (Bell et al., 2006). RDTs detect a variety of proteins, including $P$. falciparum histidine-rich protein 2 (PfHRP2) and $P$. falciparum lactate dehydrogenase (PfLDH), both specific to $P$. falciparum, and also Plasmodium LDH (pLDH) and aldolase, enzymes shared by the 5 human pathogenic Plasmodium species (Moody et al., 2002). RDTs for malaria are based on the detection of either histidine-rich protein 2 , produced only by Plasmodium falciparum, or parasite specific lactate dehydrogenase produced by all four species (Baker et al., 2005).

This test is simple, rapid, self-performing, qualitative, two site sandwich immunoassay, and sensitive for on the spot diagnosis of malaria (Malascan, Tulip Diagnostic kit literature). Malaria antibody detection gives information regarding exposure to malaria but do not differentiate between present and past infection (Hitendra Singh et al., 2000). Hence it is not useful for diagnosis in acute case of malaria.

RDTs specific for detecting antibodies against Plasmodium-derived antigens also exist. Antibodies against parasite antigens appear within days to weeks after infection and persist for months or even years. Therefore, malaria antibody tests can detect parasite infection even at low parasitemia (< 50parasites $/ \mu 1)$. Malaria antibody test, thereby, is useful in malaria endemic areas, in which light infection and/or re-infection of parasites are common. They are also helpful in testing patients who have recently been treated for malaria but their diagnosis is questioned as the microscopic examination of this case can result in a false-negative. Owing to these characteristics of malaria antibody tests, they are used for the screening of donated bloods in blood banks to prevent transfusion-transmitted malaria (Drakeley et al., 2005; Vinetz et al., 1998). 


\section{Materials and Methods}

This study was conducted in Department of Microbiology, Dhiraj General Hospital, during 6 months period (from July 2016 to December 2016) at SBKS MI and RC, Piparia, DistVadodara.

\section{Samples}

A total of 100 clinically suspected cases of malaria were included in the study. $5 \mathrm{ml}$ of whole blood was collected into the collection tube containing EDTA by venepuncture from patients presenting clinically with fever with chills and rigor and other suggestive symptoms of malaria (Thomas et al., 2012).Confirmation of diagnosis of malaria was done using three available techniques, the criteria for confirmation of positivity was positive by any of the three following methods.

\section{Thick and thin blood smears}

The peripheral smears stained with Giemsa stain For preparation of blood films, thoroughly cleaned $26 \mathrm{~mm} \times 76 \mathrm{~mm}$ glass slides. Thick and thin blood smears were prepared as per the standard method. The smears were stained with Giemsa stain. Thick smears were reported negative after examination of 200-300 oil immersion fields with no parasites observed; a thin smear was given negative when no parasites were observed in 200 oil immersion fields(David et al., 2002: $4^{\text {th }} \mathrm{ed}$ ).

Detection of antigen: Samples of all these 100 were also tested for Malarial Antigen detection by Rapid Diagnostic Test (RDT) using Malascan Pan/pf kit. The malaria pLDH /HRP2 test kit contains a membrane strip, which is precoated with two antibodies as two separate lines across a test strip. One monoclonal antibody is pan specific to $\mathrm{pLDH}$ of the Plasmodium vivax and the other line consists of a monoclonal antibody specific to the HRP2 of the Plasmodium falciparumspecies. $5 \mu 1$ of whole blood was added into the sample well. Then two drops of assay buffer were added into the buffer well. The result was read in 20 min.

Detection of antibody: Also these samples were tested for detection of antibodies against malarial parasite using One step malaria pf/pv antibody test kit (Accucare One step Malaria $\mathrm{Pf} / \mathrm{Pv}$ antibody test)as per manufacturer's instruction. The Malaria Antibody test contains a membrane strip, which is precoated with two monoclonal antibodies as two separate lines across a test strip. One monoclonal antibody (test line 1) is specific to the P.falciparum MSP and another monoclonal antibody (test line 2) is CSP of Plasmodium species (P.falciparium, P.vivax, P.malariae, P.ovale) The conjugate pad is dispensed with monoclonal antibodies conjugated to the colloidal gold, which are specific to P.falciparium MSP and pan specific to the CSP of other Plasmodium species.

\section{Statistical analysis}

Data was statistically analyzed using Medcalc -sensitivity and specificity, positive and negative predictive value testing.

\section{Results and Discussion}

A total of 66 samples were found to be positive out of these male patients were 44 and female patients were 22, using any of the three methods (Giemsa stain, RDT for antigen and antibody test). In the present study out of total 100 samples tested, 66, 63 and 58 were positive by Giemsa staining, RDT for antigen and One step Malaria Antibody test respectively (Table 1). 
On examination of positive samples; Giemsa staining, RDT for antigen and one step malaria antibody test could confirm the diagnosis of $P$. vivax in $33(50 \%), 30(48 \%)$, $17(29.3 \%)$ and that of $P$. falciparum in 26 (39.3\%), 17 (27\%), 15(26\%) and mixed infection $(P$. falciparum $+P$. vivax) in 7(10.6\%), 16(25.3\%), 26(45\%) respectively.

While sensitivity of Giemsa staining, RDT for antigen and one step malaria antibody test for detection of $P$. vivax was $100 \%, 90.9 \%, 51.5 \%$ and specificity is $100 \%, 100 \%, 100 \%$ and for
$P$. falciparum sensitivity of Giemsa staining; RDT for antigen and One step Malaria Antibody testwas $100 \%, 65.3 \%, 57.6 \%$ and specificity of $100 \%, 100 \%, 100 \%$, respectively (Table 2 and 3). Thus the sensitivity of Giemsa staining, RDT for antigen and One step Malaria Antibody test for detection of Plasmodium spp was $100 \%, 95.4 \%, 87.8 \%$ and specificity was $100 \%$ for all the three. The RDT for antigen had a sensitivity and negative predictive value higher than the malaria antibody test.

Table.1 Comparison of various methods for detection of different species of malaria parasite

$$
(\mathrm{N}=100)
$$

\begin{tabular}{|c|c|c|c|c|c|c|c|c|c|}
\hline \multirow[t]{3}{*}{ S. No } & \multirow[t]{3}{*}{ Method } & \multicolumn{8}{|c|}{ Malarial Parasite Species } \\
\hline & & \multicolumn{2}{|c|}{ None } & \multicolumn{2}{|c|}{ P. vivax } & \multicolumn{2}{|c|}{ P. falciparum } & \multicolumn{2}{|c|}{$\begin{array}{l}P . \text { vivax }+P . \\
\text { falciparum }\end{array}$} \\
\hline & & $\mathbf{n}$ & $\%$ & $\mathbf{n}$ & $\%$ & n & $\%$ & $\mathbf{n}$ & $\%$ \\
\hline 1 & Giemsa $(\mathrm{N}=100)$ & 34 & $34 \%$ & 33 & $33 \%$ & 26 & $26 \%$ & 07 & $7 \%$ \\
\hline 2 & RDT Mal $\operatorname{Ag}(\mathrm{N}=100)$ & 37 & $37 \%$ & 30 & $30 \%$ & 17 & $17 \%$ & 16 & $16 \%$ \\
\hline 3 & One step Antibody(N=100) & 32 & $32 \%$ & 17 & $17 \%$ & 15 & $15 \%$ & 26 & $26 \%$ \\
\hline
\end{tabular}

Table.2 Diagnostic efficacy of different methods for malarial parasite species

\begin{tabular}{|l|l|l|l|l|l|}
\hline \multirow{2}{*}{ Method } & \multicolumn{5}{|c|}{ Diagnostic Efficacy } \\
\cline { 3 - 6 } & Sensitivity & Specificity & PPV & NPV & Diagnostic Accuracy \\
\hline For P. vivax & & & & & \\
\hline Giemsa stain & $100 \%$ & $100 \%$ & $100 \%$ & $100 \%$ & $100 \%$ \\
\hline RDT & $\mathbf{9 0 . 9 \%}$ & $100 \%$ & $100 \%$ & $95.71 \%$ & $97.0 \%$ \\
\hline One step antibody & $51.52 \%$ & $100 \%$ & $100 \%$ & $80.72 \%$ & $84.0 \%$ \\
\hline For P.falciparum & & & & & \\
\hline Giemsa stain & $100 \%$ & $100 \%$ & $100 \%$ & $100 \%$ & $100 \%$ \\
\hline RDT & $65.38 \%$ & $100 \%$ & $100 \%$ & $89.1 \%$ & $91.0 \%$ \\
\hline One step antibody & $57.69 \%$ & $100 \%$ & $100 \%$ & $87.0 \%$ & $89.0 \%$ \\
\hline $\begin{array}{l}\text { For } \boldsymbol{P} \text {. vivax+ } \\
\text { P.falciparum }\end{array}$ & & & & & \\
\hline Giemsa stain & $100 \%$ & $100 \%$ & $100 \%$ & $100 \%$ & $100 \%$ \\
\hline RDT & $100 \%$ & $90.3 \%$ & $43.7 \%$ & $100 \%$ & $91.0 \%$ \\
\hline One step antibody & $100 \%$ & $79.5 \%$ & $26.9 \%$ & $100 \%$ & $81.0 \%$ \\
\hline
\end{tabular}


Table.3 Diagnostic efficacy of different methods for malarial parasite species

\begin{tabular}{|l|l|l|l|l|l|}
\hline \multicolumn{5}{|c|}{ Method } & \multicolumn{5}{|c|}{ Diagnostic Efficacy } \\
\cline { 2 - 6 } & Sensitivity & Specificity & PPV & NPV & Diagnostic Accuracy \\
\hline RDT & $95.4 \%$ & $100 \%$ & $100 \%$ & $91.8 \%$ & $97.0 \%$ \\
\hline $\begin{array}{l}\text { One step } \\
\text { antibody }\end{array}$ & $87.8 \%$ & $100 \%$ & $100 \%$ & $80.9 \%$ & $92.0 \%$ \\
\hline
\end{tabular}

Giemsa stain:Thick smear:P.falciparum ring and gamete

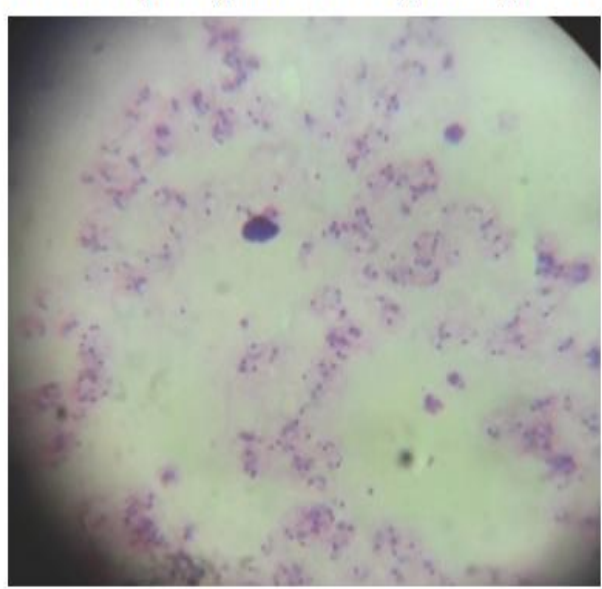

II: Rapid Malaria antigen test

1)Negative 2) P.vivax 3)Pf or mixed infection

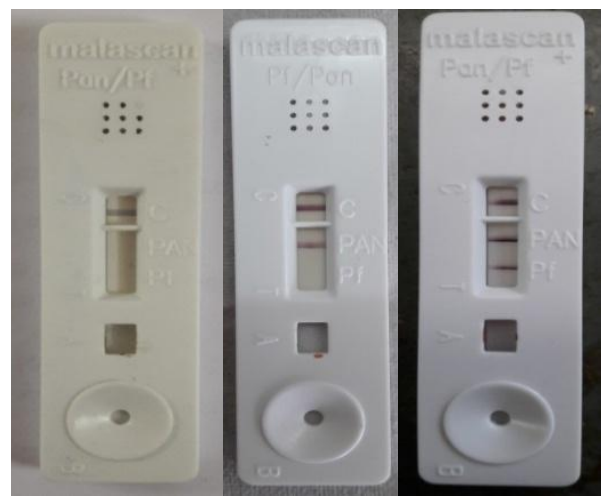

Giemsa stain:Thick smear:Pvivax late

trophozoite

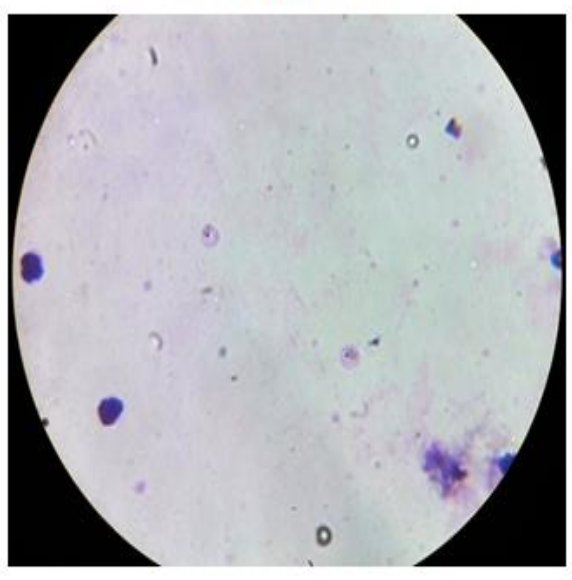

III: Malaria Antibody Detection Test
1)Negative
2)Pf
3) $\mathrm{Pv}$
4) $\mathrm{Pf}+\mathrm{Pv}$

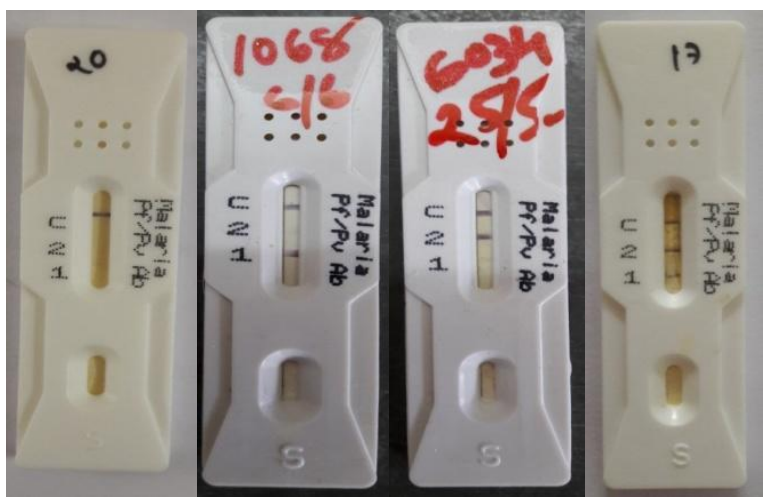



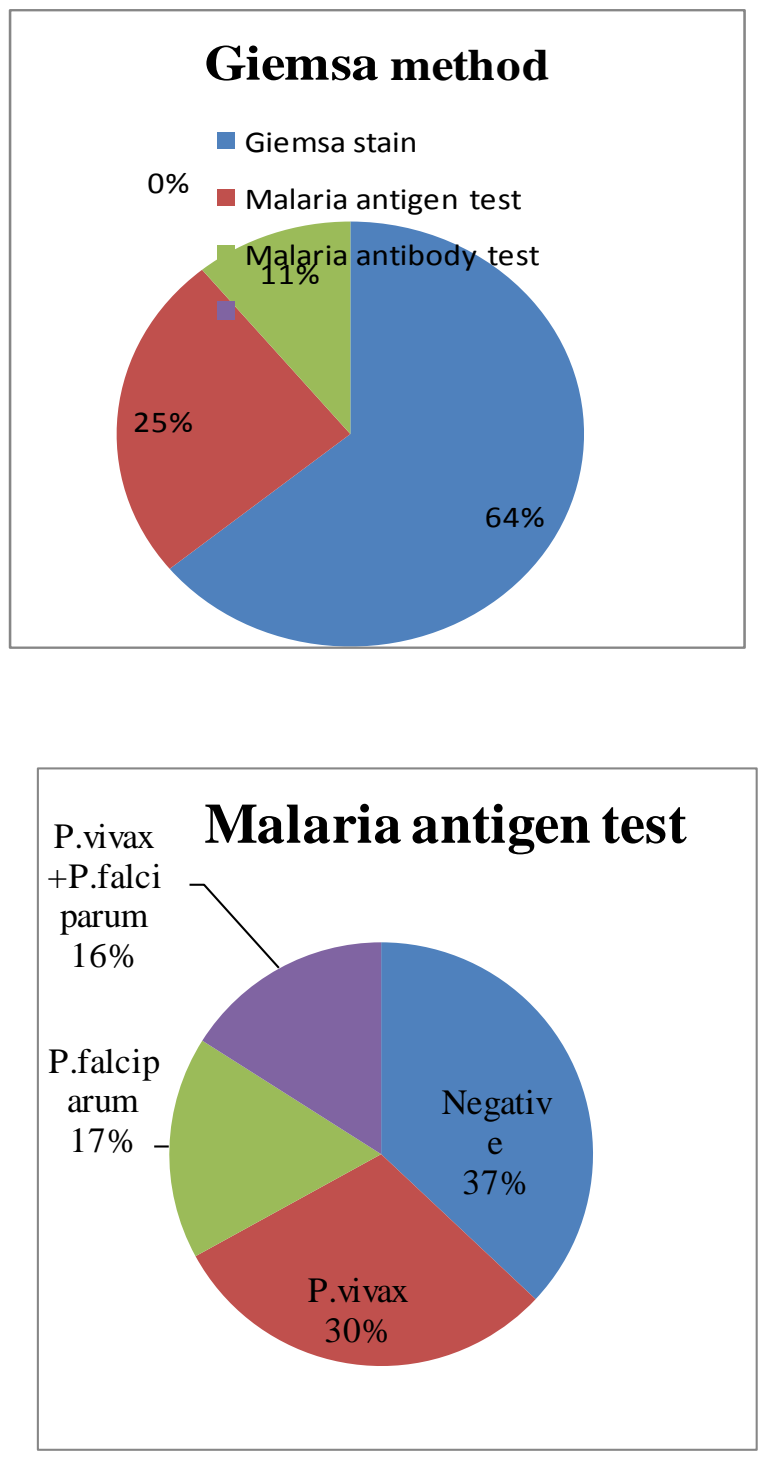

In the present study, a total of 100 clinically suspected malaria patients were included. PSMP by Giemsa stain was positive in 66 patients and 34 pts were negative. Of the total 66 malaria positive, $P$. falciparum was in $39.4 \%$, P.vivax in $50 \%$ and mixed infection in $10.6 \%$.In similar study malaria positive $P$. falciparum was in $14 \%$ and $P$. vivax in $72 \%$ detected (PrakritiVohra et al., 2014),

In present study sensitivity and specificity of Giemsa stain is $100 \%$ each. In other studies Giemsa to be $72.9 \%, 93.1 \%$ sensitive and $100 \%,>95 \%$ specific for diagnosis of malaria respectively (Gay et al., 1996 and
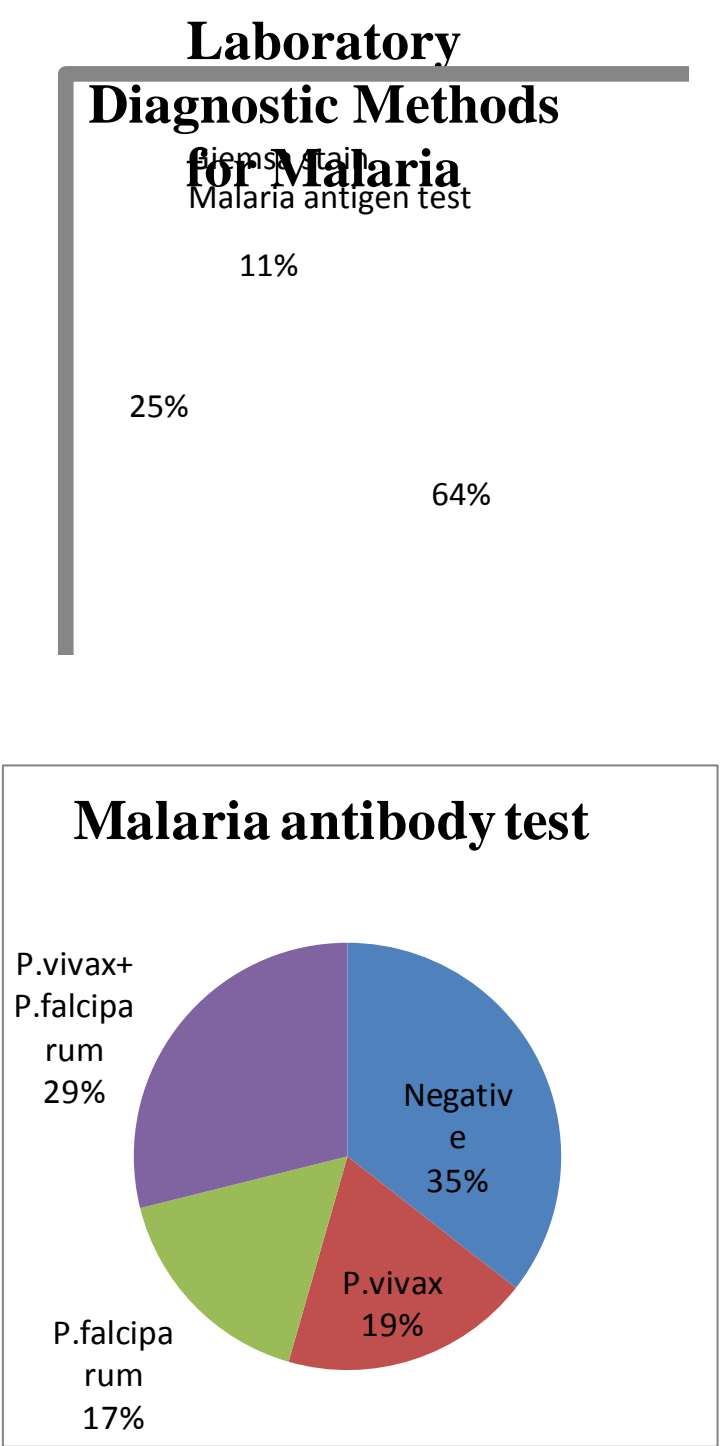

Craig et al., 1997). Malarial antigen test was positive in 63 pts and 37 pts were negative. Of the total 63 malaria positive, P. falciparum was in $27 \%, P$. vivax in $48 \%$ and mixed infection $25.3 \%$. Also 3 pts out of 37 malarial antigen negative were positive by PSMP examination. Stauffer et al., 2014, found the RDT for Malaria P.f./P.v. test to be $100 \%$ sensitive and $99 \%$ specific for $P$. falciparum, and $86 \%$ sensitive and $99 \%$ specific for $P$. vivax. Comparison with other studies Aslan et al., 2001), (Malik et al., 2006) as well as a similar study done in our department (Sucheta et al., 2013) shows the similar findings. 
Some malaria infections detected by blood film were not detected by the Accucare Malarial Antigen test. This may be explained by the fact that increased awareness of malaria among the general public as led to a rampant misuse of antimalarial drugs in inadequate doses empirically for any fever and since malascan detects pLDH which is produced only by living parasites, the blood samples tested negative by malascan may have been due to dead parasites and not yet cleared from the host or this could be due to insufficient enzyme production which occurs during early malarial infection or the patient blood samples contained parasites at concentrations below the malascan test detection level (Beatriz et al., 2012,Kakkilaya et al., 2003).

Malaria Antibody test was positive in 58 pts and 42pts were negative. Of the total 58 malaria positive, $P$. falciparum was in $25.8 \%$, $P$. vivax in $29.3 \%$ and mixed infection in $44.8 \%$ respectively. Also 34 pts out of $42 \mathrm{pts}$ negative for malaria antibody test were found to be negative for malarial Ag and PSMP examination; 5 pts out of 42 pts were positive for malarial Ag as well as PSMP examination and 3 pts out of 42 pts were positive by PSMP examination but negative by malaria $\mathrm{Ag}$. Moreover, one patient out of 58 patients who were positive for malarial antibody, tested negative for PSMP examination and malaria antigen test. This patient was sickle test positive and the patient responded to antimalarial drug.

Microscopic detection and identification of Plasmodium species in Giemsa-stained thick blood films for screening and thin blood films for species' confirmation remain the gold standard for laboratory diagnosis (Bharti et al., 2007).

In conclusion giemsa staining of peripheral smear, which is the Gold standard, as compared to existing techniques available for diagnosis of malarial parasite, still remains the best method as it demonstrates the direct evidence. But then it needs expertise whereas rapid diagnostic test for antigen detection gives quite similar results and is also accurate, saves time, can be used in fields and does not need expertise to interpret the results for $P$. vivax and for $P$. falciparum separately. For $P$. vivax and $P$. falciparum, Giemsa stain is most sensitive and has better diagnostic accuracy. For $P$. vivax, RDT is most sensitive and has better diagnostic accuracy than P.falciparum. Half of the world's population is at risk of malaria, Rapid Diagnostic Tests (RDTs) is recommended for all patients with suspected malaria before treatment is started. While using only malarial antibody test, $15 \%$ of patients with acute malaria will be missed. Malarial antibody test is useful for seroepidemiological surveys and gives information regarding exposure to malaria but does not differentiate between present and past infection and thus should not be used as a single test for diagnosis of acute malaria.

\section{References}

Aslan G, Utukanllgii M, Seyrek A, ErelOzcan. Diagnostic performance characteristics of rapid dipstick test for Plasmodium vivax malaria. MemInst Cruz Rio de janeiro 2001; 96(54): 683-6.

Baker, J., J. McCarthy, M. Gatton et al., "Genetic diversity of Plasmodium falciparum histidine-rich protein 2 (PfHRP 2) and its effect on the performance of PfHRP2-based rapid diagnostic tests," The Journal of Infectious Diseases, vol. 192, no. 5, pp. 870-877, 2005.

Beatriz EF, Iveth J González, Fanny de Carvajal, Gloria I Palma, Nancy G Saravia Mem Inst Oswaldo Cruz, Rio de Janeiro, Performance of OptiMAL in the Diagnosis of Plasmodium vivax 
and Plasmodium falciparum Infections in a Malaria Referral Center in Colombia. 2012; 97 (5) 731-35

Bell, D., C. Wongsrichanalai, and J.W. Barnwell, "Ensuring quality and access for malaria diagnosis: how can it be achieved?" Nature Reviews Microbiology, vol. 4, no. 9, pp. S7S20, 2006

Bharti, A. R., K. P. Patra, R. Chuquiyauri et al., Polymerase chain reaction detection of Plasmodium vivax and Plasmodium falciparum DNA from stored serum samples: implications for retrospective diagnosis of malaria, American Journal of Tropical Medicine and Hygiene, vol. 77, no. 3, pp. 444-446, 2007

Butler D. Time to put malaria out of control. Nature 1997; 386: 535-41.

Craig M H, Bredenkamp B L, Vaughan C H, Rossouw E J, Kelly VJ, Kleinschmidt I et al., (2002), "Field and laboratory comparative evaluation of ten rapid malaria diagnostic tests", Transactions of royal society of tropical medicine \& Hygiene, Vol. 96, pp.258-265.

Drakeley CJ, Corran PH, Coleman PG, Tongren JE, McDonald SL, CarneiroI, et al., Estimating medium- and longterm trends in malaria transmission byusing serological markers of malaria exposure. ProcNatlAcadSci U S A. 2005; 102: 5108-13.

Essential Malariology book, Edition 4th, 2002, David A Warrell, Herbert M Gilles Evaluation of different methods for diagnosis of P. falciparum malaria. Indian J Med Microbiol2006 Jan; 24(1): 49-519

Hitendrasingh G Thakor, Laboratory diagnosis of Malaria, Indian Med. Assoc. vol 98,no-10,october $2000 \mathrm{H}$. Reyburn, R. Mbatia, C. Drakeley et al., "Overdiagnosis of malaria in patients with severe febrile illness in
Tanzania: a prospective study," British Medical Journal, vol. 329, no. 7476, pp. 1212-1215, 2004

Kakkilaya BS. Rapid Diagnosis of Malaria. Lab Medicine. 2003; 8(34): 602-0834

Kyabayinze, D. J., J. K. Tibenderana, G. W. Odong, J. B. Rwakimari, and $\mathrm{H}$. Counihan, "Operational accuracy and comparative persistent antigenicity of HRP2 rapid diagnostic tests for Plasmodium falciparum malaria in a hyper endemic region of Uganda," Malaria Journal, vol. 7, article 221, 2008.

Makler MT, Palmer CJ, Ager AL. A review of practical techniques for the diagnosis of malaria. Annals of Tropical Medicine and Parasitology 1998; 92: 419-33.

Malik S, Khan S, Das A, Samantaray JC Gay F, Traore B, Zanoni J, Danis M, Blane F(1996), "Direct acridine orange fluorescence examination of blood slides compared to current techniques for malaria diagnosis", Transactions of royal society of tropical medicine \& Hygiene, Vol. 90, pp. 516-518

Manson's Tropical diseases, 22 edition, edited by Gordon C.Cook, AlimuddinI. Zumla) M. L. McMorrow, M. I.Masanja, S. M. K. Abdulla, E. Kahigwa, and S. P. Kachur, "Challenges in routine implementation and quality control of rapid diagnostic tests for malaria-Rufiji District, Tanzania," The American Journal of Tropical Medicine and Hygiene, vol. 79, no. 3, pp. 385-390, 2008.

Moody, A., Rapid diagnostic tests for malaria parasites, Clinical Microbiology Reviews, vol. 15, no. 1, pp. 66-78, 2002

Mwangi, T. W., M. Mohammed, H. Dayo, R. W. Snow, and K. Marsh, "Clinical algorithms for malaria diagnosis lack utility among people of different age 
groups," Tropical Medicine and International Health, vol. 10, no. 6, pp. 530-536, 2005.

Ninth edition, Hunter's Tropical Medicine and emerging Infectious diseases, edited by Alan J. Magill, Edward T. Ryan, David R. Hill and Tom. Solomon. Prakriti et al., 2014, Vohra, International Journal of Pharma Medicine and Biological Sciences, 2014. Topic: Comparison of peripheral blood film stained by Giemsa stain, Acridine orange staining and rapid diagnostic tests for detection of P.vivax and P.falciparum in clinically suspected cases of Malaria Prakriti Vohra et al., 2014 technique using a malaria diagnosis microscope in Myanmar", Acta Med Okayama, Vol. 56, pp. 219-222

Rosenthal, P. J., How do we best diagnose malaria in Africa? The American Journal of Tropical Medicine and Hygiene, vol. 86, no. 2, pp. 192-193,
2012

Stauffer W M, Cartwright C, Olson D, Taylor B, Bowers S H et al., (2014), "Diagnostic Performance of Rapid Diagnostic Tests versus Blood Smears for Malaria in US Clinical Practice", Vol. 58, pp. 1-8

Sucheta J Lakhani, Krunal K Shah, Pragnesh P Patel," Comparative Study of Rapid Malaria Antigen test with Giemsa Stained Peripheral Smear for Diagnosis of Malaria, IJSR, Volume 2, Issue-6, June 2013.

Thomas Jones Mackie, James Elvins McCartney, J G Collee (Eds.) (2012), Mackie \& McCartney practical medical microbiology, Churchill Livingstone, New Delhi. Vinetz JM, Li J, McCutchan TF, Kaslow DC. Plasmodium malariae infection in an asymptomatic 74-year-old Greek woman with splenomegaly. N Engl J Med. 1998; 338: 367-71.

\section{How to cite this article:}

Sangita Vasava, Sucheta Lakhani, Jitendra Lakhani and Radhika Khara. 2019. Comparison of Peripheral Blood Films Stained by Giemsa Stain, Rapid Diagnostic Tests for Antigen and Antibody for Detection of Plasmodium vivax and Plasmodium falciparum in Clinically Suspected Cases of Malaria. Int.J.Curr.Microbiol.App.Sci. 8(01): 1163-1171. doi: https://doi.org/10.20546/ijcmas.2019.801.122 